\title{
Population characteristics of Akodon montensis (Sigmodontinae) in response to habitat degradation and food availability
}

\author{
Julieta P. Sánchez-Martínez and Robert D. Owen ${ }^{2 *}$ \\ ${ }^{1}$ Departamento de Biología, Facultad de Ciencias Exactas y Naturales, Universidad Nacional de Asunción, San Lorenzo, Paraguay. \\ Email: julisanmar@gmail.com (JPS-M). \\ ${ }^{2}$ Centro para el Desarrollo de la Investigación Científica, Asunción, Paraguay, y Deptartment of Biological Sciences, Texas Tech \\ University, Lubbock. Texas, U.S.A. Email: rowen@pla.net.py (RDO) \\ * Corresponding author
}

\begin{abstract}
Small mammal populations can be affected by habitat degradation, causing changes in their abundance, density and movement. Akodon montensis, a persistent host for Orthohantavirus, is a common rodent species in primary and secondary forest habitats and is considered a generalist species. This paper analyzes how habitat degradation and resource availability affect the population characteristics of the species. Six plots were classified into three levels of degradation, with sampling conducted in June and November 2015 . After the June sampling, three plots were selected for the increase of food resources for three months, to assess how this factor affects the population. Abundance was estimated with the capture-mark-recapture method and density was estimated by dividing abundance by the effective sampling area. Home range was calculated using the Minimum Convex Polygon method, and the Maximum Distance Traveled as the longest average movement between two sampling stations where an individual was encountered. More degraded habitats supported lower average density and abundance of $A$. montensis compared to less degraded habitats. Increasing food availability led to increases in abundance in the more degraded habitats and decreases in the least degraded. Changes in home range were most evident, decreasing in the least degraded plots after the increase in resources. The sex ratio did not differ from equity in any plot, nor with respect to any of the factors studied. Population characteristics of the species are determined by several factors, including habitat quality and food distribution and abundance. If changes occur in these factors (either artificially or naturally) then movement, abundance and population density are affected in response to such changes. Although some results were not statistically significant, an apparent interaction was observed between habitat quality and resource availability, thereby influencing the abundance and density of $A$. montensis.
\end{abstract}

Las poblaciones de pequeños mamíferos pueden verse afectadas por las degradaciones en el hábitat, ocasionando cambios en la abundancia, densidad y movimiento de los mismos. Akodon montensis, un persistente hospedero para el Orthohantavirus, es una especie de roedor bastante común en hábitats de bosque primario y secundario, y es considerado como una especie generalista. Este trabajo analiza cómo la degradación del hábitat y la disponibilidad de recursos alimenticios, afectan las características poblacionales de la especie. Seis parcelas se clasificaron en tres niveles de degradación, realizándose muestreos en junio y noviembre del 2015. Luego del muestreo de junio, tres parcelas fueron seleccionadas como experimentales con el aumento de recursos durante tres meses, para evaluar cómo afecta este factor a la población, y las otras tres parcelas se mantuvieron como control sin el aumento de recursos. La abundancia se estimó con el método de captura-marca-recaptura y la densidad se estimó dividiendo la abundancia por el área efectiva de muestreo. Se calculó el área de acción mediante el método de Polígono Mínimo Convexo. Hábitats más degradados registraron un menor promedio de densidad y abundancia comparando con las degradaciones más bajas. Con el aumento de recursos, la disponibilidad de alimento generó aumentos en la abundancia de los hábitats más degradados mientras que disminuyeron en el menos degradado. Se observaron principalmente cambios en el área de acción, que disminuyeron en las parcelas menos degradadas luego del aumento de recursos. La proporción de sexos no presentó diferencias a la equidad en ninguna parcela, ni con respecto a alguno de los factores estudiados. Las características poblacionales de las especies están determinadas por factores como la calidad del hábitat, la distribución y abundancia de alimento. Cambios (artificiales o naturales) en estos factores, afectan el movimiento, la abundancia y densidad de las poblaciones. Aunque algunos resultados no fueron estadísticamente significativos, se observa cierta interacción entre la calidad del hábitat y disponibilidad de recursos, que influyen principalmente en la abundancia y densidad de $A$. montensis.

Keywords: abundance; habitat quality; home range; maximum distance moved; resource augmentation; sex ratio.

(C) 2021 Asociación Mexicana de Mastozoología, www.mastozoologiamexicana.org

\section{Introducción}

La fragmentación y transformación del hábitat han aumentado considerablemente debido al incremento de las actividades humanas, lo que afecta la diversidad, abundancia, densidad y distribución de las poblaciones silvestres. Los ensambles de pequeños mamíferos se ven ampliamente afectados por estas alteraciones, favoreciendo en gran medida a especies generalistas o de amplia distribución, y provocando a su vez un empobrecimiento de la diversidad (Cebollada-Pütz et al.2012; Santos-Filho et al. 2012; García-Estrada et al. 2015).
Esos cambios generados en el hábitat estarían afectando la ecología de hospederos naturales de patógenos zoonóticos, aumentando su incidencia y prevalencia en ciertos paisajes (Dearing y Dizney 2010), ocasionando un aumento en la abundancia y cambios en la distribución de dichas especies, incrementando así el posible contacto y transmisión de los patógenos (Suzán et al. 2008; Rubio et al. 2014).

Aunque los estudios al respecto son limitados y hasta controversiales, en los subtrópicos húmedos la precipitación parece tener un efecto menor sobre las poblaciones de hos- 
pederos de patógenos (Prist 2016), ya que se relacionan más con la estructura del hábitat (Prado 2015; Prist 2016). Cuando las alteraciones antrópicas, como por ejemplo la agricultura, enriquecen el medio ambiente para los hospederos, pareciera aumentar también su abundancia, y por lo tanto la prevalencia de los patógenos (Goodin et al. 2006; Dearing y Dizney 2010). También se generan cambios en el comportamiento y movimiento de las especies, los desplazamientos y área de acción de acuerdo con la distribución y disponibilidad de recursos alimenticios, siendo menores los cambios en aquellos hábitats con mayor disponibilidad de recursos y aumentan en hábitats más pobres (Desy et al. 1990; Batzli 1992).

La nutrición, el comportamiento espacial y la depredación se han propuesto como principales factores limitantes de la densidad poblacional de los roedores, pero no existe un acuerdo general sobre la importancia relativa de estos factores, debido a que sus interacciones no han sido estudiadas con mayor profundidad (Desy y Batzli 1989). Los cambios en las poblaciones a través del tiempo y del espacio, se producen en respuesta a las variaciones ambientales (condiciones físicas, hábitat, recursos, competidores y depredadores) y éstos a su vez se reflejan en el comportamiento y fisiología de los individuos de la población (crecimiento, reproducción, supervivencia y movimiento; Batzli 1992).

El roedor sigmodontino Akodon montensis se encuentra frecuentemente en hábitats perturbados en el Bosque Atlántico (Oliveira et al. 2014; D’Elía y Pardiñas 2015). A pesar de su carácter generalista, al ser un hospedero de Orthohantavirus es importante comprender si las alteraciones en el hábitat afectan a sus características poblacionales, para comprender su comportamiento ante los cambios.

El objetivo de esta investigación fue analizar cómo las características poblacionales (abundancia, densidad, clases de edad, proporción de sexo y área de acción) de la especie se ven afectadas por diferentes niveles de degradación del hábitat $o$ ante un cambio en la disponibilidad de los recursos alimenticios.

\section{Materiales y Métodos}

Área de estudio. La Reserva Natural del Bosque Mbaracayú (RNBM) posee 64,405 hectáreas que se localizan al noreste de la región oriental del Paraguay, en el Departamento de Canindeyú, entre los $-24^{\circ} 00^{\prime}$ y $-24^{\circ} 18^{\prime} \mathrm{S}$, y $-55^{\circ} 17^{\prime}$ y $-55^{\circ} 32^{\prime} \mathrm{O}$ (Figura 1). La misma se localiza en un área de transición entre las ecorregiones Bosque Atlántico Interior y Cerrado (FMB/BM 2005).

Diseño de muestreo. Seis parcelas fueron seleccionadas sobre la base de resultados de estudios previos, donde se encontraron roedores seropositivos para anticuerpo contra Orthohantavirus (Eastwood et al. 2018; Barreto Cáceres y Owen 2019). Se colocaron parcelas de 12 x 12 estaciones, cada estación separada por $10 \mathrm{~m}$, con dos trampas Sherman $^{T M}$ cada una colocada a nivel del suelo. Como cebo se utilizó una mezcla avena con mantequilla de maní. El esfuerzo de trampeo fue 1,440 noches-trampa por parcela por muestreo, y 17,280 noches-trampa en total.

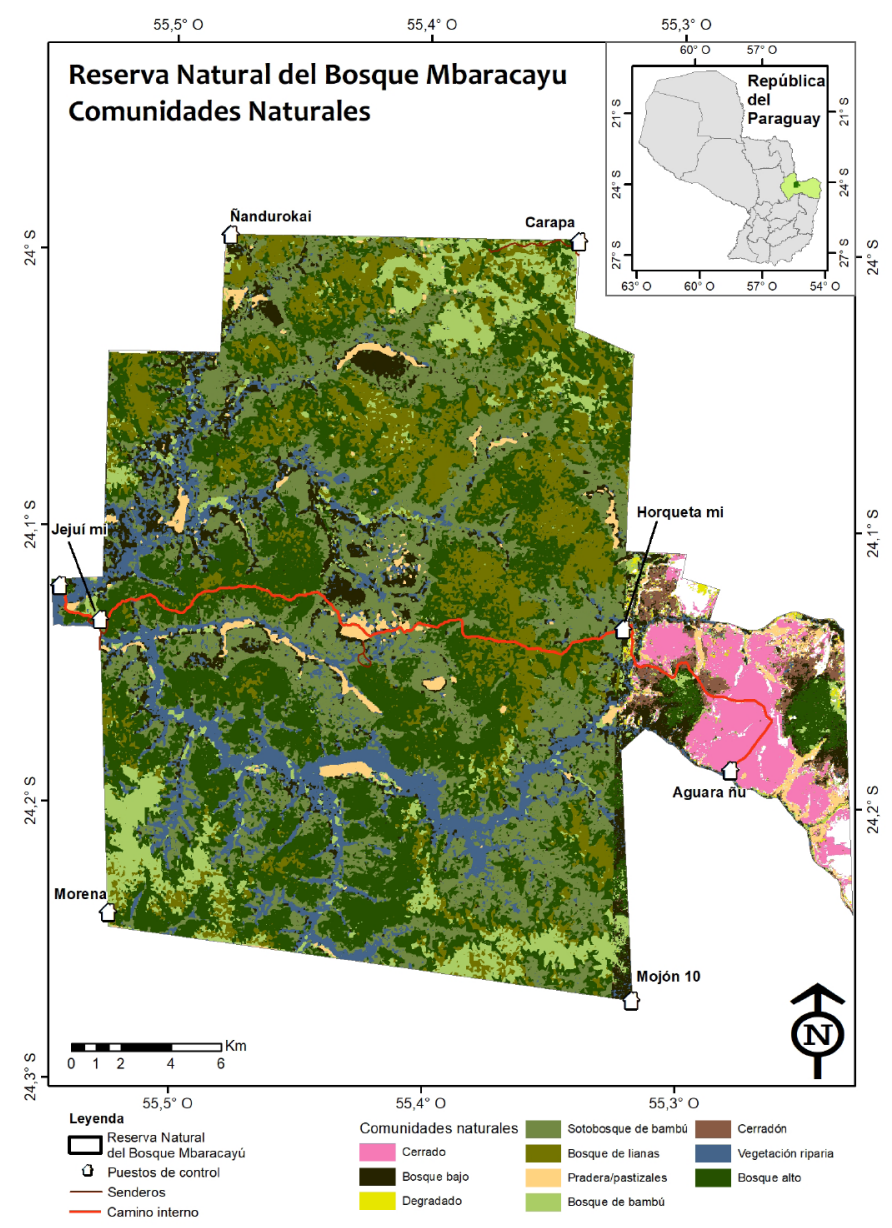

Figura 1. Ubicación de la Reserva de Biósfera del Bosque Mbaracayú (Canindeyú, Paraguay), hábitats encontrados dentro de la reserva y ubicación de las parcelas. Ubicación de la Reserva está entre $-24.000^{\circ},-24.275^{\circ} \mathrm{S},-55.322^{\circ},-55.543^{\circ} \mathrm{O}$. Coordenadas de los centros de las seis parcelas: A) $-24.1239^{\circ} \mathrm{S},-55.5048^{\circ} \mathrm{O}$. B) $-24.1412^{\circ} \mathrm{S},-55.3664^{\circ} \mathrm{O}$. C) $-24.1316^{\circ} \mathrm{S},-55.5020^{\circ} \mathrm{O}$; D) $-24.1314^{\circ} \mathrm{S},-55.4326^{\circ} \mathrm{O}$. G) $\left.-24.1306^{\circ} \mathrm{S},-55.5369^{\circ} \mathrm{O} . \mathrm{H}\right)$ $-24.1212^{\circ} \mathrm{S},-55.4651^{\circ} \mathrm{O}$. Figura modificada de Owen et al. (2018).

Las parcelas fueron evaluadas sobre la base de las características estructurales de la vegetación: porcentaje de cobertura de hierbas, porcentaje de madera en suelo, altura del dosel, árboles caídos cercanos, distancia al árbol más cercano y presencia de árboles de Citrus aurantium (una especie invasiva, indicador de degradación del bosque), medidas en cada estación. Los promedios de estos datos de cada estación se utilizaron como medidas generales para cada parcela, y fueron analizados mediante Análisis de Componentes Principales con Árbol de Recorrido Mínimo (Principal Component Analysis with Minimum Spanning Tree), hecho con NTSYSpc (Rohlf 2018). Se establecieron como indicadores de la degradación del hábitat características que se relacionan con mayor porcentaje de cobertura de hierbas, presencia de árboles de Citrus aurantium y árboles caídos cercanos, menos restos de madera en el suelo, menor altura del dosel y distancias menores entre los árboles cercanos a cada estación (Owen et al. 2019a).

Sobre la base de estos análisis citados, se designaron tres pares de parcelas de acuerdo con la calidad del hábitat, definidas en términos de degradación: menos degradado $(B$ y $H)$, medio degradado ( $A$ y $D$ ) y más degradado $(C$ y $G$ ). Tres parcelas $(H, D, y, C)$ fueron seleccionadas para el aumento experimental de los recursos alimenticios y las otras tres $(B, A$ y $G)$ per- 
manecieron como parcelas control (sin aumento de recursos). Para facilitar la comparación de varias publicaciones basadas en esta investigación de campo, las designaciones de parcela $(A, B, C, D, G, H)$ se corresponden con las de las otras publicaciones realizadas en la misma área.

Las parcelas fueron muestreadas en 2015 en dos sesiones, cinco noches consecutivas en cada una, la primera entre junio y julio, antes del aumento de recursos alimenticios, y la segunda en noviembre luego de tres meses de aumento de recursos. Para la colecta de los datos se utilizó el método de captura-marca-recaptura, donde a cada individuo se le colocó entre los hombros un Passive Integrated Transponder (microchip) subdérmico para su posterior identificación. De cada individuo capturado se registraron todos los datos estándar: identificación específica, peso, sexo, estado reproductivo y edad (adultos o subadultos, teniendo en cuenta características externas para el estado reproductivo, pelaje subadulto o adulto y el peso del individuo). Estudios previos en la misma reserva han verificado que solamente una especie de Akodon ocurre en el área. Para su identificación, se tuvo en cuenta que $A$. montensis se distingue de $A$. azarae debido a su mayor tamaño, y de A. cursor debido a la ausencia de la vesícula biliar (característica verificada en los especímenes colectados en el área en estudios recurrentes).

Aumento de recursos. El alimento seleccionado para el aumento de los recursos fue la mezcla "Super Mix" disponible como alimento para mascotas, que consiste en maíz en grano, maní con cáscara, girasol, granos balanceados y arroz en cáscara. Se diseñaron "alimentadores" (ver Figura 2), para que sólo pequeños mamíferos pudieran acceder a las semillas, y se colocaron en las parcelas correspondientes. Aunque la comida fue elegida sobre la base de pruebas de "cafetería" con Akodon, cualquier pequeño roedor tenía acceso libre a los alimentadores. Resultados sobre las características de la comunidad de los mamíferos pequeños aparecen en Barreto Cáceres y Owen (2019) y Owen et al. (2019b).

El aumento de recursos se realizó a través de la colocación de 16 estaciones de comida en un patrón uniforme, en las tres parcelas experimentales. La cantidad de alimento, $150 \mathrm{gr}$ de alimento en cada estación de comida, se calculó sobre la base de la biomasa estimada de Akodon montensis en cada parcela, basado en los resultados de los muestreos de junio-julio. Las mismas fueron vaciadas y rellenadas cada siete días durante tres meses, iniciando al finalizar el muestreo de junio-julio y terminando antes del muestreo de noviembre.

Estimación de la población. La estimación de la abundancia se calculó por el método de captura-marca-recaptura de individuos aplicando modelos que trabajan con poblaciones cerradas. El mismo se realizó con el software de estimación MARK (White 2014).

Para la estimación de la densidad poblacional se dividió la abundancia por el área efectiva de trampeo. El área

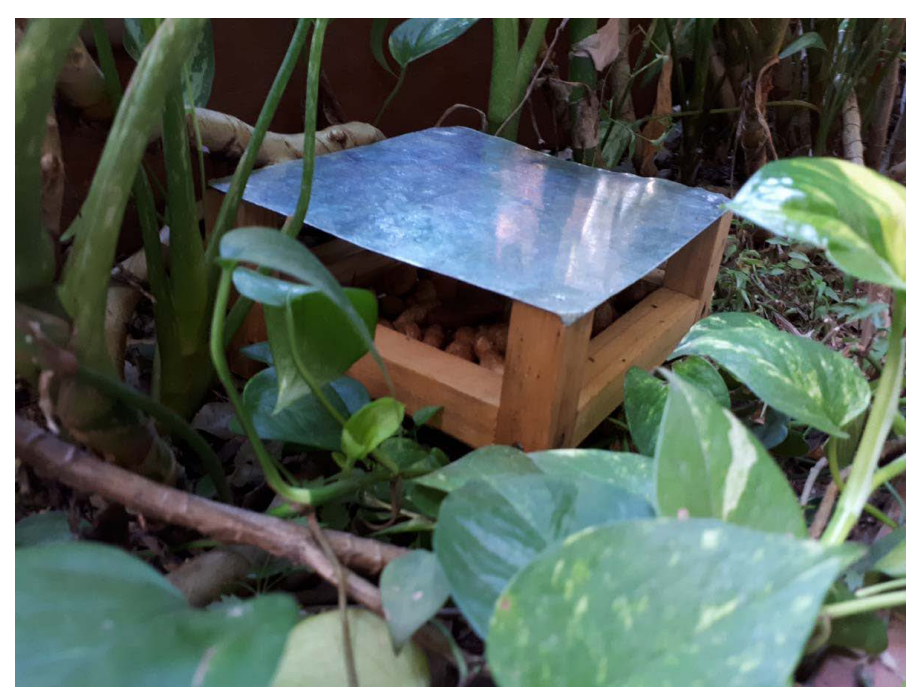

Figura 2. Alimentador. Se colocaron 16 estaciones de comida (un alimentador por estación) en un patrón uniforme, en cada una de las tres parcelas con aumento alimenticio.

efectiva se calculó para cada parcela en cada sesión de trampeo, usando la mitad del promedio de las distancias máximas recorridas por los individuos de A. montensis. Esta distancia se añadió como una franja al perímetro de cada parcela (Schnell et al. 1980), y con este dato se calculó la densidad en función al área efectiva de cada parcela y en cada muestreo. Análisis de $X^{2}$ se realizaron para determinar las diferencias de sexo, edad, las densidades y abundancias: 1) entre los diferentes niveles de degradación y 2) entre los tratamientos de disponibilidad de recursos. Para ello se utilizó el software Infostat (Infostat 2008).

Estimación del área de acción y la distancia máxima recorrida. Los cálculos del área de acción se realizaron con el software $\mathrm{R}$, usando el método de Polígono Mínimo Convexo (PCM) con el paquete "adehabitatHR" (Calenge 2011). Fueron utilizados sólo los datos de los individuos adultos que tuvieron a partir de tres capturas en puntos diferentes, con ambos sexos combinados. La Distancia Máxima Recorrida (DMR) se calculó como la distancia más larga entre dos estaciones de muestreo recorrido por un individuo (Hernández-Betancourt et al. 2003).

Se utilizó el análisis ANOVA de una vía para analizar la relación entre los tamaños de área de acción y la DMR de acuerdo con: 1) los niveles de degradación y 2) la disponibilidad de recursos. El ANOVA de dos vías se realizó para examinar la interacción entre el movimiento de los individuos, la calidad del hábitat y la disponibilidad de recursos. Para ambos análisis se utilizó el software $\mathrm{R}$ (Calenge 2011).

Los procedimientos con animales en este estudio se llevaron a cabo bajo los Permisos de Colecta Científica No. 011/2014 y 132/2015 (Secretaría del Ambiente - actualmente el Ministerio de Ambiente y Desarrollo Sostenible - Paraguay), y se siguieron las pautas del Comité de Cuidado y Uso de Animales de la American Society of Mammalogists para el uso de mamíferos silvestres en investigación y educación (Sikes et al. 2011). Todos los procedimientos con animales fueron aprobados (Aprobación 
No. 14024 - 03) por el Comité Institucional de Cuidado y Uso Animal de la Universidad de Texas Tech (IACUC-TTU), que sigue la $8^{a}$ Edición de la Guía para el Cuidado y Uso de Animales de Laboratorio (NRC 2011).

\section{Resultados}

Características poblacionales de acuerdo con los niveles de degradación. Se observaron diferencias en los datos de abundancia y densidad poblacional en los tres niveles de degradación, ambos valores fueron mayores en las parcelas con el nivel de degradación bajo (Tabla 1). Según las pruebas de $X^{2}$, tanto la abundancia como la densidad, entre niveles de degradación presentan diferencias significativas $(P=0.001$ y $P=0.002)$.

La distancia máxima recorrida fue menor en las parcelas de degradación baja, y aumenta a medida que aumenta la degradación. El área de acción fue similar entre los niveles de degradación bajo y alto, y menor en comparación con la parcela de degradación medio (Tabla 1). Sin embargo, las pruebas ANOVA de una vía realizadas para ambas medidas no presentan una diferencia significativa entre los niveles de degradación $[F(2,4)=2.752, P<0.0748]$.

La proporción de sexos no mostró diferencias en ninguna de las parcelas, ni niveles de degradación $(P>0.05)$. En las clases de edad puede observarse escasa diferencia entre los niveles de degradación, siendo similares entre los niveles de degradación bajo y alto, y levemente mayor respecto a los subadultos en las de degradación media (Tabla 1). Sin embargo, no existen diferencias significativas $(P>0.05)$.

Características poblacionales en relación con la disponibilidad de alimento. La parcela de degradación baja, posterior al aumento de recursos, registró una disminución en la abundancia, mientras que en aquellas de degradación media y alta se observó aumento de la abundancia (Tabla 1). La prueba de $X^{2}$ dio un valor de $P<0.0001$ considerándose cambios significativos en el antes y después del aumento de recursos. La densidad en la parcela de degradación baja se mantuvo similar antes y después del aumento de recursos, mientras que en las parcelas de degradación media y alta se observó un aumento considerable (Tabla 1). Sin embargo, las pruebas de $X^{2}(P=0.057)$ no mostraron diferencias significativas.
Con el aumento de recursos, la distancia máxima recorrida disminuyó en todas las parcelas, siendo más visibles en las parcelas de degradación media y alta, mientras que el área de acción disminuyó en las parcelas de degradación baja y media, y aumentó en la de degradación alta (Tabla 1). En ambos casos, los análisis no presentaron diferencia significativa (distancia máxima recorrida $P=0.109$, área de acción $P=0.933$ ).

La proporción de sexos se mantuvo igual en todas las parcelas antes y después del aumento de recursos. En cuanto a las clases de edad, se observó un aumento en el porcentaje de subadultos, luego del aumento de recursos, siendo mucha la diferencia sobre todo en las parcelas de degradación baja y alta (Tabla 1), pero sin valores de $P$ significativos. Este mismo comportamiento se observa en las parcelas control, pero las diferencias no fueron tan marcadas.

Área de acción de acuerdo con la calidad del hábitat y la disponibilidad de recursos. De los dos factores estudiados, el área de acción fue el más influenciado por la degradación del hábitat $(P=0.075)$, mientras que el aumento de los recursos alimenticios no demostró generar cambios significativos $(P=0.322)$.

Después del aumento de recursos se observó una disminución en el área de acción, incluso estratificado por nivel de degradación del hábitat, siendo más marcado en el caso del hábitat de degradación media (Figura 3), donde el nivel medio incluso tiene un descenso más marcado cruzando el nivel de degradación alta. Sin embargo, aunque esta tendencia es evidente observando los gráficos, los valores no resultaron estadísticamente significativos a través del análisis de ANOVA de dos vías (valores de $P$ : degradación $=0.314$, aumento de recursos $=0.150$, degradación*aumento $=0.546$ ) .

\section{Discusión}

Características poblacionales de acuerdo con los niveles de degradación. Abundancia y densidad. Akodon montensis es considerada generalista ya que los estudios muestran que se encuentra en distintos hábitats y asociada positivamente con diferentes factores de éstos (Goodin et al. 2009; Melo et al. 2013). Además, también está presente en bosques perturbados (Püttker et al. 2008), como se ha observado en

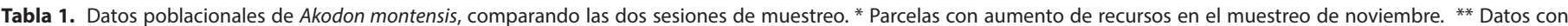
diferencias significativas.

\begin{tabular}{|c|c|c|c|c|c|c|c|c|c|c|c|c|c|}
\hline \multirow[b]{2}{*}{ Degradación } & \multirow[b]{2}{*}{ Parcela } & \multicolumn{2}{|c|}{ Abundancia } & \multicolumn{2}{|c|}{$\begin{array}{l}\text { Densidad (indiv/ } \\
\text { ha) }\end{array}$} & \multicolumn{2}{|c|}{$\begin{array}{c}\text { Distancia } \\
\text { máxima recorrida } \\
(\mathrm{m})\end{array}$} & \multicolumn{2}{|c|}{$\begin{array}{c}\text { Área de acción } \\
\left(\mathbf{m}^{2}\right)\end{array}$} & \multicolumn{2}{|c|}{$\begin{array}{c}\text { Proporción de sexos } \\
\text { (\% hembras) }\end{array}$} & \multicolumn{2}{|c|}{$\begin{array}{c}\text { Proporción } \\
\text { de edades (\% } \\
\text { subadultos) }\end{array}$} \\
\hline & & Jun & Nov & Jun & Nov & Jun & Nov & Jun & Nov & Jun & Nov & Jun & Nov \\
\hline \multirow{2}{*}{ Baja } & $B$ & 55 & 61 & 20.1 & 13.6 & 24.0 & 28.0 & 1,269 & 1,793 & 0.5 & 0.5 & 0.2 & 0.5 \\
\hline & $\mathrm{H}^{*}$ & $73^{* *}$ & $33^{* *}$ & 23.5 & 22.2 & 28.0 & 20.0 & $1,258^{* *}$ & $281^{* *}$ & 0.3 & 0.3 & 0.3 & 0.6 \\
\hline \multirow{2}{*}{ Media } & A & 87 & 77 & 29.8 & 27.2 & 26.0 & 25.0 & 2,431 & 1,425 & 0.4 & 0.5 & 0.5 & 0.6 \\
\hline & $D^{*}$ & $10^{* *}$ & $33^{* *}$ & $1.9^{* *}$ & $10.3^{* *}$ & 46.6 & $29.0^{* *}$ & 1,916 & 1,114 & 0.5 & 0.5 & 0.5 & 0.6 \\
\hline \multirow{2}{*}{ Alta } & G & 32 & 36 & 8.6 & 12.3 & 35.0 & 26.0 & 1,000 & 802 & 0.4 & 0.5 & 0.3 & 0.3 \\
\hline & $C^{*}$ & 45 & 54 & $8.6^{* *}$ & $16.6^{* *}$ & $46.0^{* *}$ & $29.5^{* *}$ & $1,183^{* *}$ & $2,757^{* *}$ & 0.5 & 0.5 & 0.2 & 0.5 \\
\hline
\end{tabular}




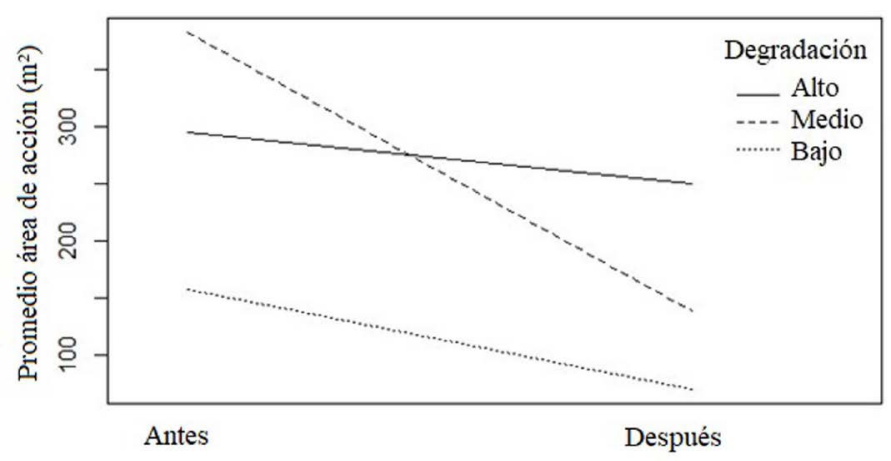

Figura 3. Gráfico de interacción entre el nivel de degradación y el aumento de recursos, mostrando los cambios en el área de acción promedio, debido al aumento de recursos.

este trabajo. Sin embargo, y si bien los análisis estadísticos no resultaron significativos, se observa una tendencia de la especie a ser más recurrente en hábitats menos degradados. La degradación estructural al nivel de microhábitat en los bosques involucra la pérdida de algunos recursos utilizados por los mamíferos (y otros animales) y que cubren requerimientos tales como sitios de anidamiento, refugio o descanso, y también sustratos para la alimentación, su pérdida puede reducir la disponibilidad de hábitats considerados de buena calidad, afectando a las poblaciones a diferentes escalas (Vergara et al. 2014).

Los resultados en este estudio demuestran que tanto la abundancia como la densidad se ven afectados por la calidad del hábitat. Esto significa que en los hábitats con menor degradación se encuentran disponibles elementos como cobertura del suelo, altura del dosel, cobertura de hierbas, entre otros, que brindan una "buena calidad" al hábitat para estos roedores. Mientras que en los hábitats con mayor degradación estaría ocurriendo una pérdida gradual y extensiva de estos elementos ocasionando una disminución de la abundancia y la densidad de las poblaciones. Los cambios en la población se reflejan en el comportamiento de los individuos, y éstos a su vez, se dan en respuesta a las diferentes variables que se encuentran en el ambiente (Batzli 1992).

Distancia máxima recorrida y área de acción. Tanto el área de acción como la DMR son medidas que reflejan la movilidad de los individuos de una población y muestran como esa población va respondiendo a los diferentes cambios del hábitat. El tamaño del área de acción (o ámbito hogareño) de las especies está determinado por varios factores, como ser la calidad del hábitat, la distribución y abundancia de alimento, la densidad poblacional, sexo y edad y la actividad reproductiva (Gentile et al. 1997; Ribble y Stanley 1998; Pires et al. 1999; Priotto y Steinmann 1999). La movilidad de las poblaciones puede estar sujeta a varios factores como la calidad del hábitat o la distribución y disponibilidad de alimento, así en hábitats con menos recursos el área de acción sería mayor comparado con hábitats más ricos en recursos (Corbalán y Ojeda 2005). En este estudio el área de acción no presentó diferencias significativas entre los diferentes niveles de degradación analizados; entonces para $A$. montensis, independiente de la degradación, el hábitat no llega a ser tan pobre para afectar de manera significativa el área de acción en estos diferentes hábitats. Esto concuerda con algunos estudios en los que algunas especies de roedores muestran adaptaciones a las diversas condiciones del hábitat (Klemann y Pelz 2006). Probablemente al ser A. montensis una especie generalista, le da cierta ventaja para mantener un área de acción en hábitats con diferentes niveles de degradación que le permitan cubrir la mayoría de los requerimientos necesarios, como alimentación, apareamiento y refugio.

Si bien no se registró una diferencia significativa para la DMR, entre los diferentes niveles de degradación, se observó una tendencia en los resultados, donde la DMR es mayor a medida que el hábitat está más degradado. Se podría considerar que en áreas degradadas los individuos necesitan moverse distancias más largas, ya sea en busca de alimento o inclusive pareja.

Proporción de sexos y clases de edad. La proporción de machos y hembras y la proporción de edades (subadultos/ adultos) se mantuvo prácticamente sin variación en los distintos niveles de degradación. Si bien las diferencias entre las clases de edad no fueron muy marcadas, se observó una mayor proporción de subadultos en los hábitats menos degradados, y una menor proporción en un hábitat más degradado. Para algunas especies de Akodon, la actividad reproductiva está asociada con la cobertura vegetal provista por el hábitat. Esto se relacionaría con los requisitos alimentarios específicos y como recurso importante para la protección, relacionadas al rendimiento de las hembras reproductivas (Escudero et al. 2014). En hábitats menos degradados la cobertura vegetal se encuentra como un factor de calidad del hábitat, lo que supondría una condición favorable para la actividad reproductiva, explicando hasta cierto punto la proporción mayor de subadultos registrados en hábitats menos degradados.

Características poblacionales en relación con la disponibilidad de alimento. Abundancia y densidad. La disponibilidad de recursos en el hábitat afecta a las poblaciones (Batzli 1992), y de acuerdo con este trabajo, las características poblacionales relacionadas a ese recurso son principalmente la abundancia y la densidad. Ambas variables aumentaron luego del enriquecimiento de recursos principalmente en las parcelas de perturbación media y alta, coincidiendo con el hecho de que, si aumentan los recursos alimenticios, aumentan la prevalencia de los roedores en el hábitat (Desy et al. 1990; Dearing y Dizney 2010). En cuanto a la parcela de baja degradación, se observó una disminución de la abundancia mientras que la densidad no sufrió modificaciones.

En las cosechas de maíz y trigo, en campos cercanos o relacionados a agroecosistemas en general, se observan efectos sobre la abundancia, movimiento y comportamiento de los roedores, ocasionando un aumento de los roedores de estos durante las cosechas (Cavia et al. 2005; Fraschina 2011; Muratore et al. 2019). Esto sugiere que mediante la suplementación y complementación del 
paisaje, se pueden mitigar los efectos de pérdida o degradación de los hábitats, y los hábitats antropogénicos que van cambiando los hábitats nativos, van compensando en cierta medida la pérdida de recursos, teniendo en cuenta sobre todo que los roedores generalistas pueden complementar sus necesidades alimentarias debido a su amplia gama alimentaria (Vergara et al. 2014).

La interacción competitiva entre todos los miembros de una comunidad es uno de los mecanismos principales por el que la disponibilidad de recursos influye en la estructura poblacional (González-Romero et al. 2005), lo que de alguna manera también explicaría lo observado en este estudio. Como se dijo anteriormente en zonas y bordes de cultivo, luego de las cosechas hay un aumento en la abundancia y riqueza de las especies de roedores, lo que influye en la composición de la comunidad (Fraschina et al. 2012; Fischer y Schröder 2014). En hábitats mayormente degradados, un aumento de recursos alimenticios beneficiaría a los roedores generalistas. Mientras que en hábitats con poca o nula degradación, esto favorecería cierta competencia debido a una mayor diversidad presente en ellas. Independientemente de este análisis, es un hecho que el hábitat poco degradado no se ve afectado por el aumento de alimento, ya que es un hábitat de buena calidad.

Distancia máxima recorrida y área de acción. En trabajos experimentales, donde se manipuló la disponibilidad de alimento, se detectó una menor área de acción como también movimientos más cortos, mientras que ambas variables aumentaron cuando no se proporcionó alimento adicional (Taylor y Quy 1978; Desy et al. 1990). Esto coincide con los resultados obtenidos en este estudio, en las parcelas experimentales con hábitats menos degradados, en promedio las distancias recorridas y los tamaños del área de acción disminuyeron luego del aumento de recursos alimentarios. Sin embargo, ocurrió lo contrario en el hábitat más degradado.

En algunos estudios se ha observado que los roedores se mueven de forma limitada y restringida, viajando distancias cortas dentro de límites de las granjas (e. g., granjas avícolas), y con mayor frecuencia en los bordes o áreas de zonas de cultivo, debido a la disponibilidad de recursos, refugio y agua que se encuentran concentrados (no ampliamente dispersos en el paisaje) y proporcionados independientemente de la temporada en esas zonas (Pocock et al. 2004; Gómez-Villafañe et al. 2008; Fraschina 2011; Muratore et al.2019). Mientras en hábitats más pobres, el tamaño del área de acción tiende a ser mayor, pues el animal necesita explorar un área mayor en la búsqueda de recursos tales como alimento (Gentile et al. 1997; Ribble y Stanley 1998).

Otros factores que pueden determinar el área de acción son el sexo o la densidad poblacional. Cuando las densidades son altas, los individuos responden restringiendo el área de acción o aumentando la superposición espacial con los otros individuos o especies (Gentile et al. 1997; Ribble y Stanley 1998). En este trabajo, en las parcelas de degradación baja no experimentales (que no tuvieron aumento de recursos) disminuyó la densidad y aumentó el área de acción, y en las de degradación alta aumentó la densidad y disminuyó el área de acción. Por otro lado, en las parcelas experimentales con menor degradación y un aumento de recursos la densidad se mantuvo similar y disminuyó el área de acción, pero en las de degradación alta ambas características aumentaron. Estos resultados estarían mostrando que existe cierta interacción entre ambas variables.

Aparentemente en las parcelas con diferentes grados de degradación en un hábitat donde no se aumenten los recursos de manera artificial, las poblaciones de A. montensis se comportan como habría de esperarse en un hábitat cualquiera que depende de los recursos alimenticios que brinda de por sí el lugar.

Proporción de sexos y clases de edad. La disponibilidad de recursos es uno de los factores que determina la conformación y el comportamiento de las poblaciones, como las clases de edad, la proporción de sexos o el movimiento, relacionadas a las variaciones ambientales en las diferentes épocas del año (Desy y Batzli 1989; Batzli 1992). En este trabajo, se observó diferencia entre las capturas de adultos y subadultos ante el aumento de alimentos. En las parcelas antes del aumento de recursos, se capturó un mayor número de adultos, mientras que después del aumento de recursos hubo mayor captura de subadultos, y también mayor captura de estos en comparación con las parcelas sin el aumento de recursos, lo que indica que el cambio de proporciones fue efecto del aumento de recursos.

Interacción entre la calidad del hábitat y la disponibilidad de recursos. El tamaño del área de acción, el movimiento y la abundancia de las especies de pequeños mamíferos están determinados por varios factores; extrínsecos como la calidad del hábitat, la distribución y abundancia de alimento, e intrínsecos como la densidad poblacional, sexo, edad, y la actividad reproductiva; además de las necesidades energéticas de cada especie (Pires et al. 1999; Priotto y Steinmann 1999). Los factores extrínsecos a nivel de microhábitat afectan las tendencias en la coexistencia de cada especie. En la mayoría de los hábitats A. montensis presenta un comportamiento dominante, sin embargo, algunas especies con las cuales $A$. montensis se ha encontrado con mayor coincidencia, muestran mayor dominio en hábitats con ciertas características, evidenciando que las respuestas de los comportamientos interespecíficos (factores intrínsecos) son determinantes para la selección del hábitat por parte de las diferentes especies en una comunidad (Owen et al. 2019b). Muchas especies de roedores comparten corredores y cruzan sus recorridos en un mismo hábitat, sin embargo, las características del mismo determinan su presencia. Por otro lado, sus movimientos son determinados no solo por el tipo de ambiente sino también por el tamaño e incluso el sexo de la especie (Maroli 2014).

En este trabajo no se analizaron detenidamente como los factores intrínsecos de la especie explicarían su comportamiento, así como tampoco se analizó de manera detenida la posible superposición con otras especies, pero se puede 
suponer que hubo algún tipo de interacción entre las especies presentes en los hábitats estudiados. Queda pendiente ver como esos otros factores influyeron también en el movimiento de la especie para este trabajo en particular.

A pesar de que los resultados estadísticos no son definitivos, se observa interacción entre los dos factores extrínsecos estudiados: la calidad del hábitat y la disponibilidad de recursos. Ambos tuvieron un impacto en la población de $A$. montensis influyendo principalmente en la abundancia y densidad. Ambas características disminuyen en hábitats degradados, pero aumentan (en el mismo hábitat degradado) con la disponibilidad de recursos alimenticios, demostrando que cualquier cambio a una pequeña escala implica un cambio en las características poblacionales del roedor. Si bien no se puede definir la interacción entre ambas variables y como eso afecta a las diferentes características poblacionales de esta especie en particular, se puede inferir cuál es la tendencia de su comportamiento. Es necesaria una mejor comprensión de la relación entre el hospedero-hábitat para prevenir escenarios futuros donde la degradación del hábitat debido a actividades que promueven la disponibilidad de recursos alimenticios, como por ejemplo la agricultura, favorezca el contacto de las personas con hospederos de enfermedades zoonóticas, como Akodon montensis.

\section{Agradecimientos}

A la Texas Tech University, por la oportunidad brindada a través de la beca ofrecida para la realización de esta investigación y al National Institutes of Health (NIH, EE.UU.) (I103053) y NIH R01 TW006986-01 a través del Programa de Ecología de Enfermedades Infecciosas de NIH-NSF. A los Profesores de la Coordinación de Postgrado, K. Núñez, A. Weiler, G. González, por el excelente trabajo llevado a cabo en todo este periodo. En especial a F. Silla por la valiosa ayuda brindada. A la Fundación Moisés Bertoni, en especial a las personas de la Reserva Natural del Bosque Mbaracayú, por ser tan buenos anfitriones y personas admirables. A las personas que nos ayudaron en el arduo trabajo de campo, E. Galeano, V. Martínez, H. Sánchez, B. Barreto, E. Ríos, A. Alfonzo, M. Sánchez, A. Rivarola y D. Bueno. A L. Rodríguez por elaborar el mapa de Figura 1. A dos revisores anónimos del artículo por las sugerencias aportadas para el enriquecimiento del trabajo. RDO agradece el apoyo del Programa de Incentivos para los Investigadores, de CONACYT-Paraguay.

\section{Literatura Citada}

Barreto Cáceres, M. B., y R. D. Owen. 2019. Relación de los pequeños mamíferos terrestres (Rodentia y Didelphimorphia) con la estructura de la vegetación en el Bosque Atlántico Interior - un análisis multivariado. Therya 10:359-369.

BatzLI, G. O. 1992. Dynamics of small mammal populations: a review. Pp. 831-850, en Wildlife 2001: populations. (McCullough, D. R., y R. H. Barrett, eds). Springer. Países Bajos. Calenge, C. 2011. Home Range Estimation in R: The AdehabitatHR Package. Office national de la classe et de la faune sauvage. Saint Benoist, Francia.
Cavia, R., I. E. Gómez Villafañe, E. A. Cittadino, D. N. Bilenca, M. H. Miño, Y M. Busch. 2005. The effect of cereal harvest on abundance and spatial distribution of Akodon azarae (Rodentia, Muridae) in Central Argentina. Agriculture Ecosystems and Environment 107:95-99.

Cebollada-Pütz, C., M. Basso, E. Ruiz de los Llanos, y M. Kufner. 2012. La fauna chaqueña de Córdoba (Argentina) afectada por la transformación agrícola. Ecología Aplicada 11:77-87.

Corbalán, V. E., y R. A. OJeda. 2005. Áreas de acción en un ensamble de roedores del desierto del Monte (Mendoza, Argentina). Mastozoología Neotropical 12:145-152.

D'Elía, G., Y U. F. J. Pardiñas. 2015. Tribe Akodontini. Pp. 140279 en Mammals of South America, Vol. 2, Rodents (Patton, J. L., U. F. J. Pardiñas y G. D'Elía, eds.). The University of Chicago Press. Chicago, EE.UU.

Dearing, M. D., y L. Dizney. 2010. Ecology of hantavirus in a changing world. Annals of the New York Academy of Sciences 1195:99-112.

Desy, E. A., y G. O. Batzl. 1989. Effects of food availability and predation on prairie vole demography: a field experiment. Ecology 70:411-421.

Desy, E. A., G. O. Batzl, y J. Liu. 1990. Effects of food and predation on behaviour of prairie voles: a field experiment. Oikos 58:159-168.

Eastwood, G., J. V. Camp, Y. K. Chu, A. M. Sawyer, R. D. Owen, X. Cao, M. K. Taylor, L. Valdivieso-Torres, R. D. Sage, A. Yu, D. G. Goodin, V. J. Martinez Bruyn, R. C. McAllister, L. Rodriguez, E. P. William, AND C. B. Jonsson. 2018. Habitat, species richness and hantaviruses of sigmodontine rodents within the Interior Atlantic Forest, Paraguay. PLoS One 13:30201307.

Escudero, P., S. Ivana, J. Polop, y M. Provensal. 2014. Environmental variables and reproductive activity in small rodents of pampean agroecosystems. Mammalia 78:23-33.

Fischer, C., Y B. SCHRöder. 2014. Predicting spatial and temporal habitat use of rodents in a highly intensive agricultural area. Agriculture, Ecosystems \& Environment 189:145-153.

FM, AND BBM. 2005. Reserva Natural del Bosque Mbaracayú. Plan de Manejo 2005-2010. Asunción - Paraguay: Fundación Moisés Bertoni para la Conservación de la Naturaleza (FMB), Banco Mundial (BM). Asunción, Paraguay.

Fraschina, J. 2011. Efectos de cambios en el uso de la tierra sobre ensambles de roedores en agroecosistemas pampeanos. Tesis Doctoral. Universidad de Buenos Aires, Buenos Aires, Argentina.

Fraschina, J., V. A. Leon, Y M. BusCh. 2012. Long-term variations in rodent abundance in a rural landscape of the Pampas, Argentina. Ecological Research 27:191-202.

García-Estrada, C., Y. A. Peña-Sánchez, y H. Colín-Martínez. 2015. Diversidad de mamíferos pequeños en dos sitios con diferente grado de alteración en la Sierra Sur, Oaxaca, México. Revista Mexicana de Biodiversidad 86:1014-1023.

Gentile, R., P. S. D'Andrea, y R. Cerqueira. 1997. Home ranges of Philander frenata and Akodon cursor in a Brazilian restinga (coastal shrubland). Mastozoología Neotropical 4:105-112.

Gómez-Villafañe, I. E., E. Muschetto, y M. Busch. 2008. Movements of Norway rats (Rattus norvegicus) in two poultry farms, Exaltación de la Cruz, Buenos Aires, Argentina. Mastozoología Neotropical 15:203-208.

González-Romero, A., L. Hernández, J. W. Laundré, E. Aragón, y J. López-Portillo. 2005. Monitoreo de dos comunidades de 
roedores en la reserva de la biosfera Mapimí, Durango, México. Pp 15-26, en Contribuciones Mastozoológicas en Homenaje a Bernardo Villa (Sánchez-Cordero V., y R. A. Medellín eds.). Instituto de Biología and Instituto de Ecología, Universidad Nacional Autónoma de México. Ciudad de México, México.

Goodin, D. G., D. E. Koch, R. D. Owen, Y. K. ChU, J. M. S. Hutchinson, y C. B. Jonsson. 2006. Land cover associated with hantavirus presence in Paraguay. Global Ecology and Biogeography 15:519-527.

Goodin, D. G., R. Paige, R. D. Owen, K. Ghimire, D. E. Koch, Y-K. CHU, Y C. B. Jonsson. 2009. Microhabitat characteristics of Akodon montensis, a reservoir for hantavirus, and hantaviral seroprevalence in an Atlantic forest site in eastern Paraguay. Journal of Vector Ecology 34:104-113.

Hernández-Betancourt, S. F., R. López-Wilchis, J. A. Cimé-Pool, y S. Medina-Peralta. 2003. Área de actividad, movimiento y organización social de Heteromys gaumeri Allen y Chapman, 1897 (Rodentia: Heteromyidae) en una selva mediana subcaducifolia de Yucatán, México. Acta Zoológica Mexicana 90:77-91.

Infostat. 2008. InfoStat versión 2008. Grupo InfoStat, FCA, Universidad Nacional de Córdoba. Córdoba, Argentina.

Klemann, N., y H. J. Pelz. 2006. The feeding pattern of the Norway rat (Rattus norvegicus) in two differently structured habitats on a farm. Applied Animal Behaviour Science 97:293-302.

MARoLI, M. 2014. Determinación de los movimientos diarios y selección de microhábitats de pequeños roedores en la Reserva Natural Otamendi, Buenos Aires. Mastozoología Neotropical 2:186-187.

Melo, G. L., B. Miotto, B. Peres, y N. C. Cáceres. 2013. Microhabitat of small mammals at ground and understorey levels in a deciduous, southern Atlantic Forest. Anais da Academia Brasileira de Ciências 85:727-736.

Muratore, M., M. de la Reta, S. Perna, A. Oggero, S. Ferrero, J. J. Polop, y M. C. Provensal. 2019. Microhabitat use by sigmodontine rodents in crop-field borders of pampean agroecosystems. Mastozoología Neotropical 26:183-189.

National Research Council of the National Academies (NRC). 2011. Guide for the care and use of laboratory animals. The National Academies Press. Washington, EE.UU.

Oliveira, R. C., R. Gentile, A. Guterres, J. Fernandes, B. R. Teixeira, V. Vaz, F. Valdez, L. Vicente, S. Da Costa-Neto, C. Bonvicino, P. D'Andrea, y E. S. Lemos. 2014. Ecological study of hantavirus infection in wild rodents in an endemic area in Brazil. Acta Tropica 131:1-10.

Owen, R. D., H. Sánchez, L. Rodriguez, y C. B. Jonsson. 2018. Composition and characteristics of a diverse didelphid community (Mammalia: Didelphimorphia) in sub-tropical South America. Occasional Papers, Museum of Texas Tech University 358:1-18.

Owen, R. D., J. V. Camp, y C. B. Jonsson. 2019a. Sigmodontine community and species responses to EI Niño and precipitation in different levels of forest degradation. Therya 10:255-265.

Owen, R. D., J. V. Camp, R. Sage, L. Rodríguez, V. J. Martínez Bruyn, R. C. MCAllister, y C. B. Jonsson. 2019b. Sympatry and habitat associations of sigmodontine rodents in a neotropical forest-savanna interface. Mammalia 84:227-23.

Pires A. S., F. A. S. Fernandez, y D. Defreitas. 1999. Patterns of space use by Micoureus demerarae (Marsupialia: Didelphidae) in a fragment of Atlantic forest in Brazil. Mastozoología Neotropical 6:39-45.

Pocock M. J. O, J. B. Searle, y P. C. L. White. 2004. Adaptations of animals to commensal habitats: population dynamics of house mice Mus musculus domesticus on farms. Journal of Animal Ecology 73:878-888.

Prado, A. F. 2015. Abundância de roedores reservatorios de hantavirus no bioma de Mata Atlántica: efeitos da estructura da paisagem e da escala de análise. Tesis de Maestría, Universidade de São Paulo, Brasil.

Priotto J. W., y A. R. Steinmann. 1999. Factors affecting home range size and overlap in Akodon azarae (Muridae: Sigmodontinae) in natural pasture of Argentina. Acta Theriologica 44:37-44.

Prist, P. R. 2016. O risco de transmissão da Hantavirose em função do clima e da estructura da paisagem. Tesis de Doctorado, Universidade de São Paulo, Brasil.

Püttker, T., R. Pardini, Y. Meyer-Lucht, y S. Sommer. 2008. Responses of five small mammal species to micro-scale variations in vegetation structure in secondary Atlantic Forest remnants, Brazil. BMC Ecology 8:1-10.

Ribble, D. O., y S. Stanley. 1998. Home ranges and social organization of syntopic Peromyscus boylii and P. truei. Journal of Mammalogy 79:932-941.

Roнlf, F. J. 2018. NTSYSpc: Numerical Taxonomy System. ver. 2.21c. Applied Biostatistics, Inc. New York, EE.UU.

Rubio, A., R. Ávila-Flores, y G. Suzán. 2014. Responses of small mammals to habitat fragmentation: Epidemiological considerations for rodent-borne Hantaviruses in the Americas. Ecohealth 11:526-533.

Santos-Filho, M., C. A. Peres, D. J. da Silva, y T. M. Sanalotti. 2012. Habitat patch and matrix effects on small-mammal persistence in Amazonian forest fragments. Biodiversity and Conservation 21:1127-1147.

Schnell, G. D., R. D. Owen, R. K. Chesser, y P. G. Risser. 1980. Populations of small mammals in north-central Oklahoma. The Southwestern Naturalist 25:67-80.

Sikes, R. S., W. H. Gannon y the Animal Care and Use Committee of the American Society of Mammalogists. 2011. Guidelines of the American Society of Mammalogists for the use of wild mammals in research. Journal of Mammalogy 92:235-253.

Suzán, G., A. Armién, J. N. Mills, E. Marcé, G. Ceballos, M. Ávila, J. Salazar-Bravo, L. Ruedas, B. Armién, y T. L. Yates. 2008. Epidemiological considerations of rodent community composition in fragmented landscapes in Panama. Journal of Mammalogy 89:684-690.

TAYLOR K. D., Y R. J. Quy. 1978. Long distance movements of a common rat (Rattus norvegicus) revealed by radio-tracking. Mammalia 42:63-71.

Vergara, P. M., A. Rivera-Hutinel, A. Farías, H. Cofré, H. Samaniego, y I. J. Hahn. 2014. ¿Cómo responden los animales del bosque a las perturbaciones antropogénicas? Pp 235254 en Ecología Forestal: Bases para el Manejo Sustentable Vol. 8 (Donoso, C., M. E. González, A. Lara, y P. Donoso, eds.). Marisa Cuneo Ediciones, Chile. Ediciones Universidad Austral de Chile. Valdivia, Chile.

White, G. C. 2014. Program MARK. Warner College of Natural Resources at Colorado State University. Boulder, EE.UU. 
Associated editor: Mónica Díaz

Submitted: January 11, 2020; Reviewed: March 9, 2020;

Accepted: July 7, 2020; Published on line: July 22, 2020. 
AKODON MONTENSIS, HÁBITAT Y RECURSOS

14 THERYA Vol. 12 (1): 5-13 